\title{
IN MARGINE AD ALCUNI NUOVI TESTI FALISCHI ${ }^{1}$
}

\author{
ROBERTO GIACOMELLI (*)
}

RIASSUNTO. - Tre nuovi testi falischi ci offrono tratti del più antico latino informale.

$* * *$

ABSTRACT. - In three new Faliscan epigraphical texts we find some features of ancient vernacular Latin.

Giovanni Colonna ha pubblicato nel 2005 un paio di interessanti testi di area sabina ${ }^{2}$ dai quali si constata la penetrazione falisca sull'altra sponda del Tevere, la presenza dell'alfabeto di V-IV secolo tipico delle due patere foied uino (pi)pafo cra carefo nonché dell'etnico Latinus.

Dell'iscrizione di Colle del Giglio, ${ }^{3}$ che "si trova su un basso calice di bucchero con piede ad anello databile verso il 500 a.C.", il Colonna dà la seguente lettura:

zo ẹko lạtinoz

Siamo in presenza di un'epigrafe di possesso "in cui a parlare è

(*) Istituto Lombardo Accademia di Scienze e Lettere, Università degli Studi di Milano.

1 La materia è più ampiamente considerata in R. Giacomelli 2009.

2 G. Colonna 2005, pp. 9-28.

3 Ibidem, p. 18. 
l'oggetto, formulata al nominativo, com'è normale nel latino arcaico, con l'eccezionale compresenza del verbo sostantivo di prima persona $\left.(z o(m) \text { per *som })^{4}\right)$. Falischi latini o laziali, dunque? L'etnico latinoz, con $-z$ per $-s$, attesterebbe piena interferenza: origini latine e non italiche, ma anche un tratto tipico ed esclusivo della grafia falisca. ${ }^{5} \mathrm{E}$ se la formula di possesso al nominativo è comune in latino arcaico, come ricorda Colonna, non lo è tuttavia in falisco in quanto già in epoca abbastanza antica, vicina a quella della nuova iscrizione, vi prevale il genitivo: eko lartos, eko kaisiosio ecc.

Rimane un dato assai probabile: il testo dovrebbe essere stato scritto "da parte di un falisco (che l'idionimo attesta di origine latina!) nella fluttuante situazione di frontiera propria di tutto il 'corridoio' del Tevere”. In termini linguistici: a fine VI secolo Falerii si dichiara latina e influenza quell'area sabina da cui ha importato peraltro numerosi tratti di lingua "bassi", che faranno da cemento della ineunte fedeltà socialtipica ${ }^{6}$ malgrado $-z$ finale $(=-s)$ sia movenza decisamente arcaica e destinata a venir meno, nel morfema in questione, durante il vigoreggiare dell'arce falisca. Fisiologica interferenza di codici a contatto, niente di sorpren-

4 Che fa pendant col plurale zot 'sunt' letto in CIE 8391 da E. Peruzzi 1967a, pp.189-191. Sul testo in questione cfr. anche la storicamente fragile seppure ingegnosa ipotesi etimologica di M. Mancini 2002, pp. 31 ss. Non entro nella questione, assai controversa, del rapporto con l'antico esum.

5 La grafia in questione, variamente interpretata, mi è parsa mutuata in falisco dall'etrusco per il tramite dell'onomastica e poi generalizzata ovunque. Che ricorra qui in un etnonimo conferma quanto scrivevo in R. Giacomelli 2006, pp. 53 ss.: "La via dell'onomastica appare in questo caso più solida che non altri ambiti di attestazione: qui nomi con $z$ furono assunti in falisco o tali e quali (zeruatronia Gia.127) ovvero falischizzati sostituendo z perfino a s etrusco originario (zaconiai Gia.109 etr. sacu); resta il fatto che il tegolo di Gia.109 in cui ricorre zaconiai (da Civita Castellana, ergo anteriore al 241 a.C.) non è riconducibile alla zona di più forte presenza etrusca dell'agro falisco, Corchiano. L'arcaicità del testo (circa IV secolo?) coinciderebbe col periodo di maggiore dinamismo di Falerii e ciò giustificherebbe, nella linea tracciata dal Peruzzi, anche questo possibile caso di pressione del falisco sull'etrusco e non viceversa. A conferma, ma col beneficio di inventario, starebbe il fatto per cui nella tomba di famiglia degli Acarcelinii le forme con $z$ si trovano nei tegoli più antichi (I,II,III), quelle con $s$ nei tegoli più recenti (IV), in cui ormai prevale il latino".

6 Alludo al periodo fra V e IV secolo in cui Falerii pare svincolarsi dallo standard arcaico capitolino e far testo, non solo nella lingua, nell'area occidentale, verso la Sabina: il concetto sociolinguistico di "fedeltà socialtipica" è mutuato da P. Trudgill 1974. 
dente per l'interlinguistica. Né è privo di interesse il fatto che $-m$ finale di $z o(m)$ sia omesso, $-z$ dell'etnonimo regolarmente trascritto.

Se l'epigrafe di colle del Giglio delinea un caso di "fluttuazione" nel senso detto, l'altro testo pubblicato dal Colonna, l'iscrizione di Foglia, attesta non solo e non tanto la presenza di Falischi fuori dell' $a$ ger Faliscus, quanto - secondo l'epigrafista - la possibile forma del relativo etnico in bocca sabina.

Il testo è inciso sopra una lastra di arenaria locale e viene datato dall'autore, entro le coordinate di altre iscrizioni consimili della zona, fra la metà del IV e il III sec. a.C. La datazione più alta deriva da una circostanza inattesa: la grafia è identica "a quella del celebre motto conviviale dipinto su due kylikes falische del Gruppo Foied, datate alla metà o poco dopo del [IV] secolo". ' Il dinamismo socioculturale di Falerii nel periodo della fedeltà socialtipica riceve conferma dall'uso di tale specifica grafia fuori del territorio propriamente falisco. Colonna motiva le proprie argomentazioni anche dal punto di vista storico e archeologico con dati eloquenti: i sepolcreti della zona circostante mostrano la prevalenza "di tombe a camera di IV sec. a.C. fornite di loculi parietali al modo falisco"; ${ }^{8}$ e inoltre Foglia si trovava sull'itinerario collegante, anche in epoca pre-romana, Falerii con Magliano, capoluogo della zona settentrionale della Sabina tiberina. ${ }^{9}$ Secondo l'epigrafista la lastra incisa doveva fare da sigillo a un loculo parietale adibito a sepoltura all'interno della camera; ne discenderebbe che il testo rechi il nome del defunto:

\section{$[---]$ ciuifahls $[\mathrm{c}---]$}

L'interpretazione non è priva di difficoltà: l'assenza di interpunzione contrasta con Gia.5 (Gia.=G. Giacomelli 1963) (foied uino...) anche se coincide con il dato dell'epigrafe di Gavios Frenaios di cui parleremo appresso. Quanto alla lingua si può trattare di una formula bimembre con

7 G. Colonna 2005, p. 12. E' possibile però una datazione leggermente più alta, perlomeno al V-IV secolo. Al momento di licenziare le bozze sono informato che il Colonna avrebbe rivisto la sua interpretazione dell'iscrizione. Non mi riesce però di reperire il contributo dell' archeologo.

8 Ibidem, p. 10.

9 Ibidem, p. 11. 
prenome e gentilizio (o cognome) oppure di una coppia di gentilizi come nelle epigrafi Gia.121.IX e 122 (assai mutile e più recenti) in cui ricorre la formula prenome+gentilizio+abbreviazione del prenome paterno; lo stesso nel testo da Pratoro /precono [/ ; /cuitene/ ; /let [/ se vale la nostra interpretazione Preconnios Quintos Neronios [bic] lectus. ${ }^{10}$ Ma l'evidenza epigrafica è modesta e le ipotesi del Colonna possono supplirla solo nei limiti del possibile: molto tentante sarebbe dividere ciui fahls [c e interpretare civis Faliscus, ma ciò presupporrebbe un testo, se pur mutilo, più ampio che indicasse anche i nomi personali e, soprattutto, lacune sui due lati di ampiezza decisamente minore; rimane il fatto che, a smentire che -ciui sia dativo, ${ }^{11}$ basterebbe la tomba degli Acarcelinii (Gia. 121) in cui ricorrono solo nominativi. Dediche al dativo sono, in falisco, isolate. ${ }^{12}$

Non meno sorprendente è il presunto etnico $f a b l s[c$ per il quale l'autore ricorre alla nota convenzione grafica umbra $/ \mathrm{Vh}=\mathrm{V}$ lunga $/{ }^{13}$ onde un * $f \bar{a} l_{s c[}[$ che, rispetto a Faliscus, deriverebbe, tra l'altro, da sincope di vocali atone provocata dall'accento espiratorio italico. In questo caso però una sequela $* f \bar{a} l s c s$ richiederebbe una lacuna meno estesa alla fine delle lettere leggibili: un parziale non liquet sembra più consigliabile senza perciò sottovalutare i pregnanti baluginii interlinguistici e socioculturali che l'iscrizione lascia intravedere. Avremmo qui, ad ogni modo, l'effetto combinato di forze centrifughe (fonetica italico-sabina) e centripete dal momento che l'espansione di Falerii si rispecchierebbe nell'ipotesi del Colonna ${ }^{14}$ - l'iscrizione pare confermarlo ancorché testis unus - che l'antico alfabeto epicorico sia stato sostituito a partire dal IV sec. da quello falisco "nel nord della Sabina tiberina".

Il secondo nuovo testo falisco è la cosiddetta iscrizione di Gavios Frenaios, studiata da J.A.Berenguer Sánchez e E.R.Luján: ${ }^{15}$ il vaso sul quale è dipinta, di cui non si conosce l'origine, è ispirato alla ceramica attica e magnogreca di V-IV secolo. L'editore ${ }^{16}$ propende per una data-

10 R. Giacomelli 2006, pp. 89 ss.

11 La discussione del Colonna su questo particolare (G. Colonna 2005, p. 13), seppure dottissima, non appare risolutiva.

12 G. Giacomelli 1963, p. 163.

13 G. Colonna 2005, pp. 14-15.

14 Ibidem, p. 17.

15 J.A. Berenguer Sánchez-E.R. Luján 2005, pp. 213-22.

16 R. Olmos Romera 2003, cit. in J.A.Berenguer Sánchez-E.R.Luján 2005, p. 213. 
zione a fine IV secolo a.C. cioè mezzo secolo avanti la distruzione dell'arce falisca, avvenuta nel 241 a.C.; potrebbe aversi qui la riprova di una caduta di tensione della fedeltà socialtipica a favore dell'odiata Urbe capitolina: come avvenuto ai Messapi, il fatto di avere in Taranto una rivale pericolosissima non impedì l'entrata di grecismi ${ }^{17}$ nell'idioma encorio, anzi ne derivò semmai il contrario grazie all'indiscutibile prestigio politico e culturale della colonia greca dorica.

Perché parliamo di sfaldamento dell'antico orgoglio? L'iscrizione, per l'epoca, dovrebbe avere lo stesso alfabeto di Gia.5, interpunzione e fattezze di lingua del cosiddetto "periodo medio". Al contrario il testo

cauiosfrenaiosfaced

mostra, quanto alla lingua, tratti più che arcaici semmai di incipiente adeguamento al latino: la conservazione di $-s$ finale (divergente dal falisco medio: ci saremmo aspettati cauio frenaio) è sia dei più antichi testi falischi e romani sia degli Elogia Scipionum capitolini, di poco successivi; una norma instabile ma già codificata se comporta le ben note oscillazioni grafiche, presenti pure in alcune varietà di latino periferico. Forza centrifuga dunque rispetto alla fedeltà socialtipica, parrebbe. $\mathrm{Ci}$ torneremo a proposito di faced.

In pieno accordo con la "cultura" falisca sembra invece l'iconografia del vaso - immagini satiresche - come suggeriscono i due linguisti spagnoli: ${ }^{18}$

¿Puede relacionarse la imagen de este vaso con ese ambiente pre-teatral en el que, en territorio itálico, a finales del s. IV a.C., podrian haberse llevado a cabo determinadas formas de representación, quiza de origen cultual o festivo, mediante coros de sátiros?

Probabile forza centripeta, all'incontro: una testimonianza, questo vaso, della tradizione fescenninica all'origine del teatro romano come ne parla Livio? Satiresca, non a caso, anche l'iconografia di Gia.5: intra vasculum Bacchus et Semele osculantes, extrinsecus inter palmites

17 Comunque debbano essere valutati numerosi grecismi tarentini sono presenti nelle iscrizioni messapiche. Cfr. R. Giacomelli 1979.

18 J.A.Berenguer Sánchez-E.R.Luján 2005, p. 221. 
ephebus nudus cum tymphano bacchans et duae feminae palliatae rapraesentantur (Herbig, CIE, ad tit.). E dionisiaca parrebbe essere ${ }^{19}$ anche l'iscrizione Gia.3.

La grafia suscita qualche dubbio: oltre a non coincidere con quella delle due note patere "Foied", presenta, secondo l'analisi paleografica di J.A.Berenguer Sánchez-E.R.Luján, ${ }^{20}$ alcuni tratti insoliti per l'epoca cui viene ascritta. In particolare la notazione della vibrante, in certo modo addirittura extra-falisca stando alla tabella degli alfabeti di G. Giacomelli: ${ }^{21}$ potrebbe pensarsi a un espediente per segnalare l'evanescenza della vibrante etimologica studiata da Mancini? ${ }^{22} \mathrm{Ne}$ adombra la realtà il gentilizio clipeaio dell'epigrafe Steinhardt, di pari datazione, su cui torneremo appresso, nei confronti del poco più recente (e "irrigidito", evidentemente) clipeario Gia. 121 IX.

Altri grafemi suscitano perplessità: $\mathrm{N}$ corrisponde a quello latino, di direzione destrorsa; A non è né quello arcaico né rispecchia il "gruppo Foied"; S infine non è, come pure ci si potrebbe attendere dato l'orientamento scrittorio generale, di tipo arcaico e nelle patere "Foied" non è attestato.

Tornando alla lingua, il gentilizio frenaios con -ai- e non -ae-oppure $-\bar{e}-$ rinforza l'impressione di un penchant per lo standard latino, confermato, non a caso, dal gentilizio latinaio Gia. $63 .{ }^{23}$ Il dittongo era sentito, evidentemente, come più "alto" anche se Pratoro a metà del II secolo attesta prētod con il monottongo; inutile ripetere che una menzione magistratuale poteva trascinare la percezione di una veneranda arcaicità indigena.

Resta da discutere faced. Dopo tanta copia di item discrepanti, la forma verbale ${ }^{24}$ - indichi l'artefice o il dedicante - si rivela indice sicuro della pur disgregantesi fedeltà socialtipica; la forma concorda con

19 Almeno secondo la nota interpretazione di E. Peruzzi 1967b, pp. 113-133.

20 J.A.Berenguer Sanchez-E.R.Luján 2005, pp. 215-16.

21 G. Giacomelli 1963, p. 30.

22 Cfr. M. Mancini 2002, pp. 34-40.

23 Cfr. anche G. Giacomelli 1963, p. 118. Del resto una donna non falisca, bensì romana è menzionata nell'arcaica epigrafe Gia.3, col nome di rufia: la forma locale sarebbe stata di sicuro * rōfia.

24 Arduo pensare (come ritengono, opportunamente, J.A.Bereguer Sánchez-

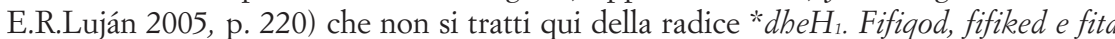
(da *dheigh-) sono, ex professo, mots techniques della coroplastica falisca. Cfr. R. Giacomelli 2006, pp. 27 ss. 
keset di Pratoro nell'adeguamento allo standard latino sicché l'inventario aggiornato del falisco non consente più di affermare che i perfetti sono tutti a raddoppiamento come scrivevo nel $1978^{25}$ sulla base della documentazione precedente ai testi di Pratoro nonché alle epigrafi di Gavios Frenaios e Steinhardt.

E' il caso di rilevare, peraltro, che il vocalismo radicale di questo perfetto rientra perfettamente nella tradizione falisca: cuncaptum, peparai, aciptum, haraspex ecc., trasparenza paradigmatica, assenza di indebolimento; tratto squisitamente "orale", che permane nel primo latino letterario, precedente a una salda standardizzazione. ${ }^{26} \mathrm{E}$ il vocalismo radicale di facet, calcato su fak- del presente, dell'iscrizione Steinhardt conferma tale stato di cose.

L'iscrizione Steinhardt, resa nota da R. Wallace, ${ }^{27}$ è dipinta sotto la base di un bel vasetto ceramico a forma di gallo di foggia e stile tipici di fine IV sec. a.C., di gusto espressionistico "laziale" e popolaresco. Lingua e grafia sono in pieno accordo con la datazione dell'oggetto parlante. Purtroppo il fatto di non conoscere il luogo di provenienza non aiuta anche se si può supporre che si tratti di prodotto dell'arce falisca (Civita Castellana) realizzato all'incirca 50 anni prima della distruzione ad opera dei Romani come si può inferire da alfabeto e interpunzione che, salvo il grafema lunato per $c$, coincidono nell'insieme con quelli del "gruppo Foied"; la trascrizione di Wallace è la seguente:

oufilo : clipeaio : letei : fileo : met : facet

25 In R. Giacomelli 1978, p. 63.

26 Su tali dati v. ad es. R.Giacomelli 1996, pp.95, 97, 106. I rapporti, veri o presunti, fra questi perfetti falischi (e romani) e il Fhefhaked della Fibula Prenestina non toccano, a mio parere, la presente ricognizione. Vari autori ne danno conto in modi differenti: v. ad es. M. Mancini 2009. I riflessi diatopico-diastratici veri o presunti che in varietà arcaiche paiono consonare con Probo vanno ora riveduti alla luce degli studi di M. Mancini 2007a e M. Mancini 2007b. Peraltro il De Simone (in C. De Simone 2006, pp. 159-175), che si schiera -dopo M. Mancini 2009- per l'autenticità della fibula, definisce a p. 173 "latinisch" (i.e. "non romano") il tipo faced; peraltro, svolgendo il tema del presunto rapporto diacronico fra Fhefhaked e faced sostiene (a p. 174) che le due forme potevano essere varianti diastratico-diafasiche. Il che si può pensare, a nostro avviso, più per l'opposizione tra la forma con vocale lunga etimologica (fécit urbano) e quella periferica (faced) nell'abito di un eventuale diasistema del latino romano usato da inquinate loquentes.

27 R. Wallace 2004, pp. 175-82. 
L'interpretazione dell'editore si perde nei meandri dello schleicherismo senza dare adeguato rilievo ai tratti di possibile valore interlinguistico: falisco e latino di Roma non erano - men che meno a fine IV secolo - monadi conchiuse in se stesse e impermeabili reciprocamente. ${ }^{28}$

I tratti di lingua sono, perlopiù, quelli della fedeltà socialtipica, che però, lo vedremo qui sotto, appare in via di sfaldamento a favore dello standard romano: $-s$ finale dei personali non è segnato; oufilo, già noto più che altro come gentilizio-patronimico ${ }^{29}$ (es. oufilio Gia.71, 140) e, assieme al prenome cauios ${ }^{30}$ di tradizione onomastica locale; a pieno titolo del falisco medio anche fileo con $-e-$ per $-i$ - né davvero appare sorprendente il già noto clipeaio con il dileguo, qui, dell'ormai evanescente antica vibrante. La formula patronimica con "filius e il genitivo" ${ }^{31}$ è di sapore latino in luogo dei più antichi derivati in -io- ed è già presente in iscrizioni da Civita Castellana, anteriori dunque (di quanto?) al 241 a.C.

Molto più decisamente però parlano a favore di un adeguamento allo standard capitolino ormai in corso $\mathrm{i}-t$ finali di met e facet: se nel verbo, come nell'epigrafe di Gavios Frenaios (faced), risalta la solita socialtipica utilizzazione del tema $f a k$ - per analogia sul presente e trasparenza paradigmatica, $-t$ finale di entrambe le forme è emblematico e coincide con keset di Pratoro rispetto al $-d$ atteso, arcaico sia in falisco sia in latino. ${ }^{32}$ Quanto al pronome $m e t^{33}$ è probabile trattarsi di ipercorrettismo: $-d$ finale veniva già percepito, come a Pratoro un secolo appresso, non in linea con lo standard capitolino, ${ }^{34}$ troppo encorio e "rustico". Del che si ha conferma, specularmente, nelle note ipercorrezioni con sonora per sorda dell'iscrizione dei cuochi specie dal momento che, via dalla morfologia, i Falischi romanizzati di metà II secolo conservano comunque la sorda in keset $\sim$ lat. gessit.

28 Cfr. R. Giacomelli 2006, pp. 52 ss.

29 G. Giacomelli 1963, pp. 176-177.

30 Ibidem, pp. 182-183.

31 Ibidem, pp. 245-246.

32 R.Wallace 2004, p.177 s'ingarbuglia in un'analisi comparativa e ricostruttiva assai poco utile ai fini di un'efficace e realistica valutazione interlinguistica.

33 Per il quale R.Wallace 2004, p. 182 invoca l'ipotesi di una ristrutturazione sillabica.

34 E lo scriba potrebbe anche avere "conguagliato" verso un'unica "modernità" le finali di pronome e verbo. 
Un chiaro parallelo nell'interventismo diastratico si ha nel latino tardo: è evidente il valore sociolinguistico "antichizzante" di $-d$ (=- $t$ secondario) se in una lettera di Claudio Terenziano ${ }^{35}$ di epoca volgare alla riga 16 si legge inquid per inquit, sicuramente non frutto di sandhi perché seguito da quod con sorda iniziale; alle righe 22-23 reliquid (=relīquit) pure seguito da sorda (con).

Forze centripete dunque, e di tradizione socialtipica in regresso, ma anche centrifughe verso uno standard romano che andava facendosi strada quanto il potere politico dell'Urbe.

35 Ph. Baldi 1999, p. 238. 


\section{BIBLIOGRAFIA}

Baldi Ph, The Foundations of Latin, Berlin-New York, de Gruyter, 1999.

Berenguer Sánchez J.A., Luján E.R., La nueva inscripción falisca de Cavios Frenaios, “ZPE”, 153 (2005), pp. 213-22.

Colonna G., Questioni tiberine: Foglia e l'etnico dei Falisci in dialetto sabino, "Annali Fondaz. Museo Faina" 13, (2005), pp. 9-28.

de Simone C., Falisco faced Latino arcaico vhevhaked: la genuinità della Fibula prenestina e i problemi connessi, "InL", 29 (2006), pp. 159-75.

Giacomelli G., La lingua falisca, Firenze, Olscki, 1963.

Giacomelli R., Problemi di storia linguistica del latino dialettale I. Ricerche falische, Firenze, La Nuova Italia, 1978.

Giacomelli R., I grecismi del messapico, Brescia, Paideia, 1979.

Giacomelli R., Storia della lingua latina, Roma, Jouvence, 1996 .

Giacomelli R., Nuove ricerche falische, Roma, Il Calamo, 2006.

Giacomelli R., Alcune nuove iscrizioni falische: forze centripete o centrifughe?, ACME 62, 2009, pp.117-133.

Mancini M., Tra linguistica ed ermeneutica: nuove acquisizioni falische, "InL", 25 (2002), pp. 31 ss.

Mancini M., "Appendix Probi". Correzioni ortografiche o correzioni linguistiche?, in F. Lo Monaco-P. Molinelli (edd.), L'Appendix Probi”.Nuove ricerche, Firenze, Ed. Galluzzo, 2007, pp. 65 ss.

Mancini M., Strutture morfoprosodiche del latino tardo nell'Appendix Probi, "RLR", 71 (2007), pp. 425 ss.

Mancini M., Il perfetto latino tra continuità e discontinuità: faciō, fēci, fefaked, in A. Ancillotti - A. Calderini (acd), L'umbro e le altre lingue dell'Italia antica - Atti del I Conv. Int. sugli antichi Umbri - Gubbio, 20-22 settembre 2001, Perugia, Jama, 2009, pp. 67-96.

Olmos Romera R., Cratera de columnas de Cavios Frenaios, in P. Cabrera (ed.), La Colleción Várez Fisa en el Museo Arqueológico Nacional, Madrid 2003, [cit. in J.A.Berenguer Sánchez-E.R.Luján, p. 213].

Peruzzi E., Un documento di ius sepulcri falisco, "Klio", 2 (1967), pp.189-91.

Peruzzi E., L'iscrizione falisca delle sociai, "PP”, 22 (1967), pp. 113-33.

Trudgill P., The Social Differentiation of English in Norwich, Cambridge, CUP, 1974.

Wallace R., A Faliscan Inscription in the M. and J. Steinhardt Collection, "ZPE", 149 (2004), pp. 175-82. 